\title{
A SEARCH FOR PRESOLAR ORGANIC MATTER IN METEORITE
}

\author{
Jongmann Yang and Samuel Epstein
}

Division of Geological and Planetary Sciences, California Institute of Technology, Pasadena 91125

\begin{abstract}
The $\mathrm{D} / \mathrm{H}$ ratios and the ${ }^{13} \mathrm{C} /{ }^{12} \mathrm{C}$ ratios of acid-insoluble organic matter of 4 meteorites, Ochansk (H4), Plainview (H5), Gladstone (H6) and Odessa (IA), were measured. $\delta D$ values for hydrogen extracted by stepwise combustion were negative, down to $-280 \%$. $\delta^{1} \mathrm{C}$ values were also negative except in the case of the carbon coming off at the highest temperature steps for Plainview and Odessa meteorites. The concentrations of ${ }^{13} \mathrm{C}$-rich carbon were 3-5 orders of magnitude smaller than those found in Murchison meteorite, suggesting that relic grains of stellar condensates were mostly destroyed in the meteorites examined.
\end{abstract}

\section{Introduction}

Hydrogen isotope studies of acid residues from various meteorites suggested that organic matter of high $\mathrm{D} / \mathrm{H}$ ratio is probably originated from interstellar organic molecules and is present in large quantities in some of the unmetamorphosed meteorites [Smith and Rigby, 1981; Robert and Epstein, 1982; Yang and Epstein, 1983a]. SD values of such organic matter were above $1,000 \%$ in most cases and up to about $10,000 \%$ in some cases. Types 4-6 ordinary chondrites and differentiated meteorites [Yang and Epstein, 1983a] contained organic matter whose $\delta \mathrm{D}$ values were similar to terrestrial hydrogen, between $-200 \%$ and $200 \%$, except Kelly (LL4) whose $8 \mathrm{D}$ value was $380^{\circ} \% \delta^{\circ} \delta^{13} \mathrm{C}^{\circ}$ values of the organic matter had a much narrower range of $-31 \%$ and $-12 \%$ [Briggs, 1963; Smith and Kaplan, 1970; Robert and Epstein, 1982; Swart et al., 1982; Yang and Epstein, 1982, 1983a]. The $\delta^{13} \mathrm{C}$ variation of about $20 \%$ in carbon samples of metamorphosed meteorites is probably an indication of isotopic fractionation processes due to a loss of carbon during metamorphism [Yang and Epstein, 1983a]. When Murchison meteorite (CM2: carbonaceous chondrite of petrologic type 2) samples which were treated with $\mathrm{HCl}-\mathrm{HF}$ and with various oxidizing chemicals were combusted by stepwise heating, the $\mathrm{CO}_{2}$ gas extracted at temperatures above $800^{\circ} \mathrm{C}$ gave $8^{13} \mathrm{C}$ values up to $1500 \%$ [Kerridge, 1983; Swart et al., 1983; Yang and Epstein, 1983b, 1984]. Swart et al. [1983] considered that this ${ }^{13} \mathrm{C}$-rich carbon was originated from red giant star materials. Assuming that all types of meteorite were originally made up of similar material, the degree of elimination of the D-rich hydrogen and the ${ }^{13} \mathrm{C}$-rich carbon should be an indication of the degree of metamorphism for petrologic types 4-6 ordinary chondrites and differentiation for iron meteorite.

\section{Experimental Procedures}

In this study we used three bronzite chrondrites $(\mathrm{H})$ and an iron meteorite of group IA - Ochansk (H4), Plainview (H5), Gladstone (H6) and Odessa (IA). Whole rock meteorite samples were treated with various chemical reagents following the procedure described by

Copyright 1985 by the American Geophysical Union.

Paper number 4L6399.

0094-8276/85/004L-6399 \$03.00
Yang and Epstein [1983b, 1984] and Alaerts et al. [1979]. Two sets of samples were prepared. The first set of samples, labeled CFO, are the bulk meteorites which were treated successively by $10 \mathrm{M} \mathrm{HCl}, 9 \mathrm{M} \mathrm{HF}-1 \mathrm{M} \mathrm{HCl}$, $70 \% \mathrm{HClO}_{4}, 90 \%$ fuming $\mathrm{HNO}_{3}, 30 \% \mathrm{H}_{2} \mathrm{O}_{2}$ and $3 \mathrm{M}$ $\mathrm{NaOH}-0.4 \mathrm{M} \mathrm{NaOCl}$ (all at $100^{\circ} \mathrm{C}$ except $\mathrm{HF}-\mathrm{HCl}$ which was at $23^{\circ} \mathrm{C}$ ), and were washed in distilled water and then in acetone. The yields of the residues ranged between 0.37 and $1.03 \%$ (Table 1). A terrestrial shale sample was also treated in a similar manner to assure that any isotopic fractionations due to our procedures would be revealed in this way.

The second set of samples, labeled CFOP, are the CFO samples which were treated further with boiling $70 \% \mathrm{HClO}_{4}$ to remove organic hydrogen and to concentrate heavy carbon by removing organic carbon with low $\delta^{13} \mathrm{C}$ values (see Yang and Epstein, 1984 for more details of chemical effects). The resulting yields by weight ranged between 0.0053 and $0.32 \%$ (Table 1). We obtained 10 acid residue samples from 4 meteorites and a shale. Odessa iron meteorite contains large black spherical inclusions. We separated out some of these inclusions during the early stage of chemical treatments for separate analyses.

Gases $\left(\mathrm{H}_{2}, \mathrm{CO}_{2}, \mathrm{SO}_{2}, \mathrm{~N}_{2}\right)$ were extracted in a quartz vacuum system which was designed to minimize the blank of the system. Samples were wrapped with Pt foil and all samples were loaded at the same time. The main system consists of several fingers for copper oxide (provides oxygen), uranium (converts $\mathrm{H}_{2} \mathrm{O}$ to $\mathrm{H}_{2}$ gas), cold liquids (separates gases), $\mathrm{Pd}-\mathrm{Ag}$ (passes $\mathrm{H}_{2}$ gas) and a resistant heater (heats samples stepwisely with $\pm 5^{\circ} \mathrm{C}$ accuracy). Gases $\left(\mathrm{N}_{2}, \mathrm{CO}_{2}, \mathrm{SO}_{2}\right)$ were collected by a Toepler pump which is connected to the main quartz system by a metal bellow valve which is the only valve in the system. Every part of the system was heated thoroughly above $-600^{\circ} \mathrm{C}$ before every sample run.

TABLE 1. The data of total concentrations of carbon and the ratios of $\mathrm{H} / \mathrm{C}, \mathrm{N} / \mathrm{C}$ and $\mathrm{S} / \mathrm{C}$ in the two kinds of acid residues of H4-6 \& IA meteorites and shale.

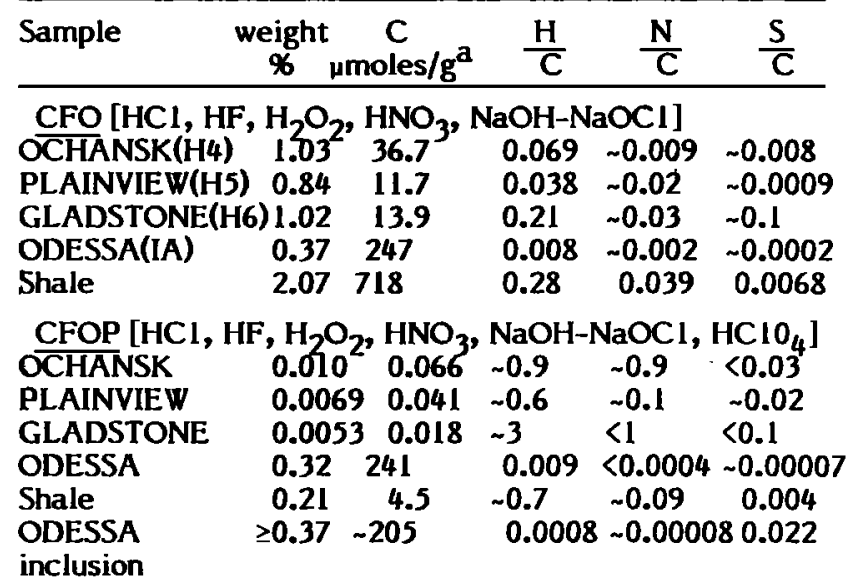

${ }^{a}$ The concentration is given in $\mu$ moles per gram of bulk samples of meteorites and terrestrial shale. 
TABLE 2. The concentrations and the isotopic data of $H$ and $C$ released by stepwise oxidation - pyrolysis of acid residues of four meteorites and a terrestrial shale.

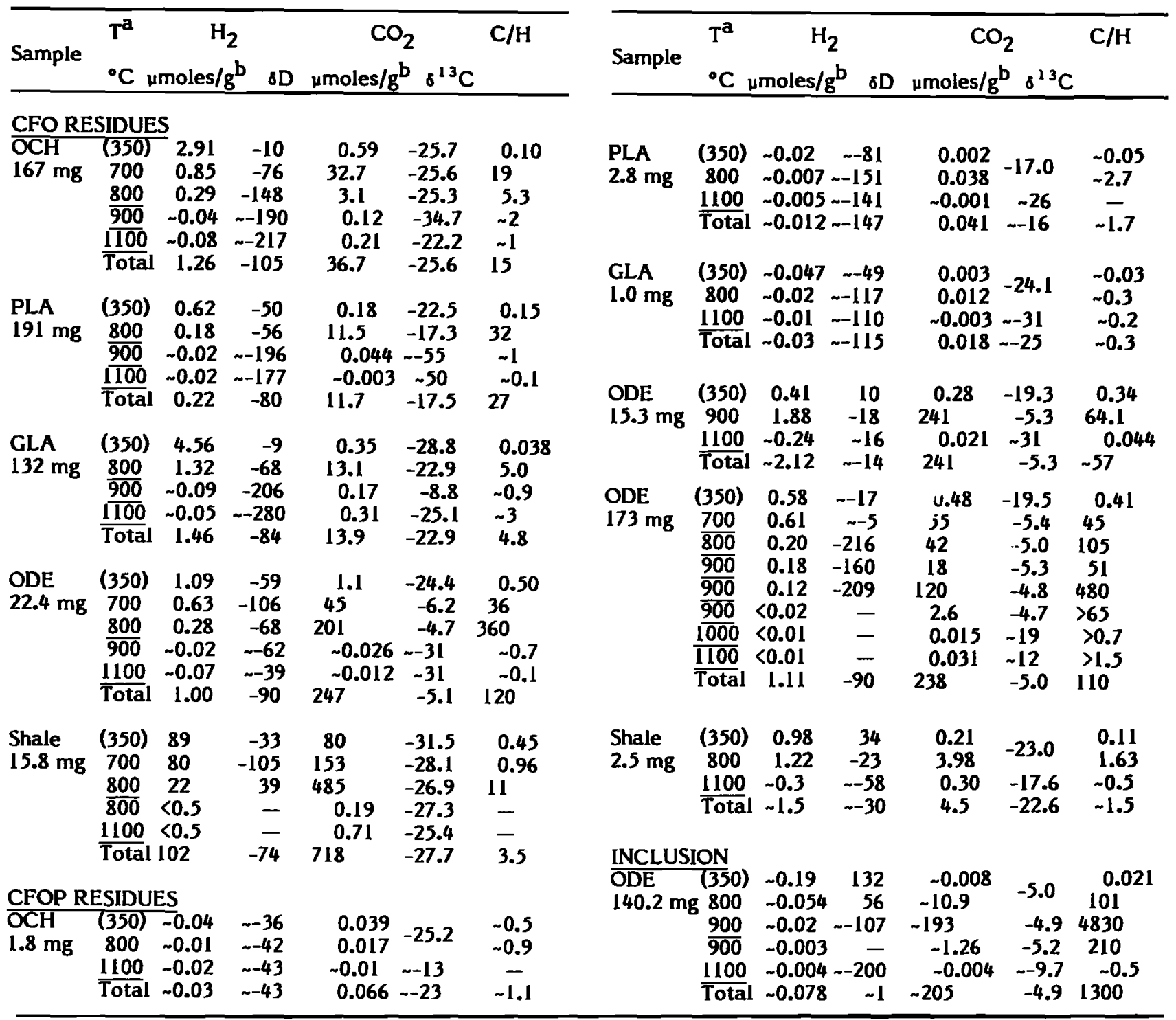

${ }^{a_{T}}$ in parentheses represents the heating step without oxygen. Samples were heated for 1-3 hours at each heating step except underlined steps where samples were heated for 4-16 hours.

byields are given in $\mu$ moles per gram of bulk samples.

Incidentally this was the identical system used with the study of Murchison samples. The blanks were especially low in carbon, nitrogen and sulfur, $0.03 \mu$ moles each. Hydrogen and carbon gas samples were analyzed by standard stable isotope ratio mass spectrometers and reported in usual $\delta$ notations. $\delta$ is the deviation in isotopic ratios with respect to standards in parts per thousand $(\%)$. The standards for hydrogen and carbon are standard mean ocean water and PDB, respectively.

\section{Results and Discussion}

Table 1 shows the percentage yield of the CFO and CFOP residues and the concentration of carbon in the residues. For the three $\mathrm{H}$ chordrite samples boiling the CFO residues with $70 \% \mathrm{HClO}_{4}$ resulted in a loss of more than $99.6 \%$ of the total carbon and more than $99 \%$ of the total weight. The perchloric acid, therefore, removed most of the material (chromite, spinel and carbon) and left a small amount of very oxidationresistant material. Table $I$ also shows the hydrogen, nitrogen and sulfur concentrations in the samples relative to carbon for comparison. A very small amount of the nitrogen and sulfur remained in the residues after treatment, $\leq l \mu \mathrm{mole} / \mathrm{g}$ for CFO and $<0.1 \mu \mathrm{mole} / \mathrm{g}$ for CFOP, so that isotopic measurements were not made for these gases. Carbon contents in Odessa meteorite samples are somewhat surprising. The weight percentages of carbon in the two residues are $81 \%$ (CFO) and 90\% (CFOP). Virtually no carbon in CFO Odessa sample was lost by perchloric acid treatment.

Table 2 shows the isotopic data and the concentrations of hydrogen and carbon released by stepwise combustion of the acid residue samples. Note that the first heating step, $350^{\circ} \mathrm{C}$, was straight pyrolysis in absence of oxygen gas. Under this condition, we 
found that terrestrial hydrogen could be degassed but the indigenous organic hydrogen of the samples is more stable in absence of $\mathrm{O}_{2}$ and comes off at above $350^{\circ} \mathrm{C}$. We consider the $350^{\circ} \mathrm{C}$ fraction of hydrogen as of terrestrial origin.

$\delta D$ values for the total hydrogen of meteorites are $-105 \%$ to $-79 \%$ for CFO samples and $-147 \%$ to $-14 \%$ for CFOP samples. The ranges of $\delta \mathrm{D}$ values of hydrogen from stepwise heating are $-280 \%$ to $-39 \%$ for CFO samples and $-216 \%$ to $-5 \%$ for CFOP samples. These $\delta D$ values are quite similar to the $\delta D$ values of $\mathrm{HCl}-\mathrm{HF}$ residues from metamorphosed and differentiated meteorite samples studied in Yang and Epstein [1983a]. The $\delta D$ values and the concentrations of hydrogen per gram of CFO acid residue samples, 26270 umoles/g, fit well in the plot of $\delta D$ vs. hydrogen concentrations in Yang and Epstein [1983a]. However, the concentrations of the hydrogen are very low and the $\delta D$ values of this hydrogen and the $\delta D$ of the inorganic meteorite hydrogen $(-110 \%)$ are similiar [Yang and Epstein, 1983a]. These results might suggest that the high temperature history of these meteorites allow the direct or indirect equilibration of the hydrogen in the organic matter with the inorganic hydrogen formed in the beginning of the solar system formation [Yang and Epstein, 1983a].

The range of $\delta^{13} \mathrm{C}$ values of total carbon in the CFO and CFOP samples of $\mathrm{H}$ chondrites is between $-26 \%$ and $-16 \%$. If the different carbons given out at different temperatures are considered, this range expands to between $-55^{\circ} \%$ and $+50 \%$. The $\delta^{13} \mathrm{C}$ value of total carbon for $\mathrm{CFO}^{\infty}$ and CFOP samples of Odessa iron meteorite is $-5^{\circ} \%$. The range of $\delta^{13} \mathrm{C}$ values of carbon from all temperature steps is between $-31 \%$ and $+31 \% . \delta^{13} \mathrm{C}$ values of the carbon released at different temperatures for a given sample are often constant if we exclude carbons from the lowest and the highest temperature steps. This is best shown in Odessa CFOP sample and demonstrates that the gas extraction procedure effectively separated different carbon components. A terrestrial shale sample was chemically treated the same way as the meteorites. The gas analyses of the residues CFO and CFOP showed no unusual isotopic values for both $\delta \mathrm{D}$ and $\delta^{13} \mathrm{C}$, indicating that the chemistry applied to the meteorites and the gas extraction procedure do not significantly change the isotopic values of the meteorites by isotopic fractionations from chemical and distillation processes.

While the carbon samples of Ochansk and Gladstone meteorites all gave $\delta^{13} \mathrm{C}$ less than $-8 \%$, carbon of Plainview and Odessa meteorites released at temperatures above $900^{\circ} \mathrm{C}$ gave $\delta^{13} \mathrm{C}$ values of 12 to $50 \%$ These positive $\delta^{13} \mathrm{C}$ values are not as large as those found in high temperature release carbon of Murchison acid residues. However, it is quite possible that they originated from the same source because the sample preparations were almost identical. Also, the residues consist of similar material (chromite, spinel and organic polymer) and the isotopic patterns from stepwise combustion are the same.

The $\mathrm{C} / \mathrm{H}$ ratios (Table 2) of CFO residues of three $\mathrm{H}$ chordrites are 4.8 to 27. CFO and CFOP samples of Odessa iron meteorite showed unusually high $\mathrm{C} / \mathrm{H}$ ratios of 110 to 120. Perhaps the carbon in this Odessa meteorite was formed by decomposition of organic matter during differentiation process unlike the other three meteorites. The existence of the high positive $\delta^{13} \mathrm{C}$ values in Odessa meteorite may possibly give information about the thermal history of the parentbody from which Odessa originated by the degree of homogenization of the total carbon and hydrogen.
We may compare the concentrations of the ${ }^{13} \mathrm{C}$-rich carbon of the Murchison residues with those of the 4 meteorites we studied here. If the concentrations of the carbon with positive $\delta^{13} \mathrm{C}$ values are compared, Murchison residues contain 0.1 to 4.0 umoles per gram of bulk meteorite sample and the residues of Plainview and Odessa contain 0.001 to $0.046 \mu$ moles per gram of bulk meteorite sample. Therefore, Murchison contains about two orders of magnitude more carbon of positive $\delta^{13} \mathrm{C}$. It may be more appropriate, however, to compare the concentrations of heavy carbon by assuming the same ${ }^{13} \mathrm{C}$ value. Previously we have used the maximum $8^{13} \mathrm{C}$ value observed, $1500 \%$, as a heavy carbon component [Yang and Epstein, 1983b]. Assuming there are two carbon components, a low $\delta^{13} \mathrm{C}$ value of $-30 \%$ and a high $\delta^{13} \mathrm{C}$ value of $+1500 \%$, the Murchison samples contained 1.3 to $66 \mathrm{ppm}$ of $1500 \% \delta^{13} \mathrm{C}$ carbon component. Similarly, we could calculate the concentrations of such a ${ }^{13} \mathrm{C}$-rich carbon component in Plainview and Odessa residues. They contain 0.3 to 2 and 4 to $6 \mathrm{ppb}$ of $1500 \%{ }^{13} \mathrm{C}$ carbon component (Table 3). Therefore, the two meteorites contain 3 to 5 orders of magnitude less heavy carbon compared to Murchison meteorite.

Metamorphism temperature for $\mathrm{H} 4-6$ chondrites is estimated to be about $600-830^{\circ} \mathrm{C}$ [Dodd, 1981 ; Lingner, Huston, and Lipschutz, 1984; Olsen and Bunch, 1984].

TABLE 3. The $\delta \mathrm{D}$ values and the concentrations of carbon of $1500 \%, \delta 13 \mathrm{C}$ in acid residue samples of meteorites.

\begin{tabular}{|c|c|c|c|}
\hline Sample & $\begin{array}{c}\delta D^{d} \\
\%\end{array}$ & $\begin{array}{c}\text { heavy carbon } \\
\text { ppm }\end{array}$ & Ref. \\
\hline \multicolumn{4}{|c|}{ MURCHISON, CM2 } \\
\hline $\begin{array}{l}\mathrm{H}_{3} \mathrm{PO}_{4} \\
2 \mathrm{C} 10 \mathrm{f} \\
2 \mathrm{C} 10 \mathrm{~m}\end{array}$ & & $\begin{array}{l}2.2 \\
3.5 \\
0.6\end{array}$ & $\begin{array}{l}\mathbf{a} \\
\mathbf{a} \\
\mathbf{a}\end{array}$ \\
\hline $\mathrm{CF}$ & 607 & 30 & b \\
\hline CFOc & 2560 & 8.6 & b \\
\hline CFOf & 952 & 37 & b \\
\hline CFOfN & 301 & 2.8 & b \\
\hline CFP & 214 & 1.3 & b \\
\hline $\mathbf{P}$ & -86 & 2.6 & b \\
\hline PCF & - & 1.3 & b \\
\hline CFS & 772 & 66 & b \\
\hline \multicolumn{4}{|c|}{ ALLENDE, CV3 } \\
\hline BB & & 0.7 & $\mathbf{a}$ \\
\hline BG & & 0.5 & a \\
\hline BIB & & - & a \\
\hline \multicolumn{4}{|c|}{ OCHANSK, $\mathrm{H}_{4}$} \\
\hline CFO & -105 & - & C \\
\hline CFOP & -43 & $<0.004$ & c \\
\hline \multicolumn{4}{|c|}{ PLAINVIEW, H5 } \\
\hline $\mathrm{CFO}$ & -80 & -0.002 & c \\
\hline CFOP & -147 & -0.0003 & c \\
\hline \multicolumn{4}{|c|}{ GLADSTONE, H6 } \\
\hline CFO & -84 & - & c \\
\hline CFOP & -115 & $<0.0007$ & C \\
\hline \multicolumn{4}{|c|}{ ODESSA, IA } \\
\hline CFO & -90 & -0.004 & c \\
\hline CFOP & -90 & $\sim 0.006$ & c \\
\hline
\end{tabular}

aSwart et al. [1983].

bYang and Epstein [1983b, 1984].

CThis work.

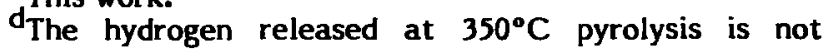
included in the summation of the total hydrogen for this $8 \mathrm{D}$. 
The cooling rates of type 4-6 ordinary chondrites and iron meteorite at above $-600^{\circ} \mathrm{C}$ are much higher than those at lower temperatures [Pellas and Storzer, 1981; Taylor and Scott, 1984]. Therefore, it may be possible that a major thermal isotopic effect occurred during cooling at, say, $600^{\circ} \mathrm{C}$ to $500^{\circ} \mathrm{C}$. The duration of that cooling period is about <2 to $14 \mathrm{myr}$ for $\mathrm{H} 4-6$ [Pellas and Storzer, 1981] and 0.25 to 22 myr for iron meteorites [Pellas and Storzer, 1981; Narayan and Goldstein, 1983].

Perhaps the thermal history of about $600^{\circ} \mathrm{C}$ for an order of 1 myr of the 4 meteorites we studied here was severe enough to destroy the anomalous $D$ and ${ }^{13} \mathrm{C}$ isotopic values of hydrogen and carbon by homogenization. The difference in $\delta^{13} \mathrm{C}$ values of total carbon between Odessa and the other three $\mathrm{H}$ chondrites is about $20 \%$ and such a value could be interpreted as a consequence of a thermal isotopic fractionation. However, the thermal history was mild enough or the mechanisms involved in homogenization slow enough for some meteorites such as Plainview and Odessa to preserve some heavy carbon even though the amount is small. All meteorites but Ochansk are finds. However, this fact does not relate with any of the trend we observed. More quantitative and meaningful constraints could be made when we understand the physical and chemical environment of the meteorite parent body more accurately.

\section{Conclusions}

Table 3 summarizes the $\delta \mathrm{D}$ values and the concentrations of the heavy carbon of $1500 \% \delta^{13} \mathrm{C}$ found in the acid residues of meteorites studied so far. Clearly CFO and CFOP samples lack the deuterium-rich organic matter formed in the interstellar medium. The concentrations of the ${ }^{13} \mathrm{C}$-rich carbon in these samples are 3-5 orders of magnitude lower than acid residues of two carbonaceous chondrites, Murchison (CM2) and Allende (CV3). Such deficiencies could be due to the metamorphism for $\mathrm{H4-6}$ and the differentiation processes for iron meteorites. Indigenous isotopic patterns could be erased during such high temperature episodes by isotopic homogenization between ${ }^{3} \mathrm{C}$-rich carbon and normal carbon in organic matter and carbonates.

The absence of high $\delta D$ and the small enrichments in the $\delta^{13} \mathrm{C}$ suggest the loss of hydrogen and carbon and the homogenization of the remaining fraction of these elements during metamorphism. The small residual $\delta^{13} \mathrm{C}$ enrichment may or may not be indicative of survival of originally high $\delta^{13} \mathrm{C}$ carbon $(-1500 \%)$ and thus these meteorites may have been void of ${ }^{13}{ }^{\circ} \mathrm{C}$-rich carbon prior to metamorphism. More detailed analyses of the residues may contribute to the answer to this alternative.

Acknowledgments. This work was supported by NASA grant NGL 05-002-190. Contribution 4154 of the Division of Geological and Planetary Sciences.

\section{References}

Alaerts, L., R.S. Lewis, and E. Anders, Isotopic anomalies of noble gases in meteorites and their origins - IIl. LL-chondrites, Geochim. Cosmochim. Acta, 43, 1399-1415, 1979.

Briggs, M.H., Evidence of an extraterrestrial origin for some organic constituents of meteorites, Nature, 197, 1290, 1963.

Dodd, R.T., Meteorites, Pp. 90-97, Cambridge University, New York, 1981.

Kerridge, J.F., Isotopic composition of carbonaceouschondrite kerogen: evidence for an interstellar origin of organic matter in meteorites, Earth Planet. Sci. Lett., 64, 186-200, 1983.

Lingner, D.W., T.J. Huston, and M.E. Lipschutz, Mobile trace elements and thermal histories of H4-6 chondrites. Comparison with L4-6 chondrites, Meteoritics, 19, in press, 1984.

Narayan, C., and J.I. Goldstein, A major revision of iron meteorite cooling rates-An experimental study of the growth of the Widmanstatten pattern, Meteoritics, 18, 360-361, 1983.

Olsen, E.J., and T.E. Bunch, Equilibration temperature of the ordinary chondrites: A new evaluation, Geochim. Cosmochim. Acta, 48, 1363-1365, 1984.

Pellas, P., and D. Storzer, ${ }^{244} \mathrm{Pu}$ fission track thermometry and its application to stony meteorites, Proc. R. Soc. Lond. A, 374, 253-270, 1981.

Robert, F., and S. Epstein, The concentration and isotopic composition of hydrogen, carbon, and nitrogen in carbonaceous meteorites, Geochim. Cosmochim. Acta, 46, 81-95, 1982.

Smith, J.W., and D. Rigby, Comments on D/H ratios in chondritic organic matter, Earth Planet. Sci. Lett., $54,64-66,1981$.

Smith, J.W., and I.R. Kaplan, Endigeneous carbon in carbonaceous meteorites, Science, 167, 1367-1370, 1970.

Swart, P.K., M.M. Grady, and C.T. Pillinger, Isotopically indistinguishable carbon phases in the Allende meteorite, Nature, 297, 381-383, 1982.

Swart, P.K., M.M. Grady, C.T. Pillinger, R.S. Lewis, and E. Anders, Interstellar carbon in meteorites, Science, 220, 406-410, 1983.

Taylor, G.J., and R.D. Edward, A quantitative look at chondrite metamorphism, Meteoritics, 19, in press, 1984.

Yang, J., and S. Epstein, On the origin and composition of hydrogen and carbon in meteorites, Meteoritics, 17, 301-302, 1982.

Yang, J., and S. Epstein, Interstellar organic matter in meteorites, Geochim. Cosmochim. Acta, 47, 21992216, 1983a.

Yang, J., and S. Epstein, Interstellar organic matter in meteorites, Meteoritics, 18, 429-430, 1983b.

Yang, J., and S. Epstein, Relic interstellar grains in Murchison meteorite, Nature, 311, 544-547, 1984.

S. Epstein and J. Yang, Division of Geological and Planetary Sciences, California Institute of Technology, Pasadena, CA 91125.
(Received November 5, 1984; accepted November 28, 1984.) 